\title{
Circulación de obsidiana en Chubut, Patagonia Central, Argentina: uso de las materias primas extra-regionales como indicadores de movilidad e interacción entre grupos cazadores recolectores
}

\author{
Analia Castro Esnal* \\ Cecilia Pérez de Micou** \\ Charles R. Stern***
}

\begin{abstract}
CASTRO ESNAL, A.; PÉREZ DE MICOU, C.; CHARLES R. STERN, Ch.R.
Circulación de obsidiana en Chubut, Patagonia Central, Argentina: uso de las materias primas extra-regionales como indicadores de movilidad e interacción entre grupos cazadores recolectores. R. Museu Arq. Etn., São Paulo, n. 21, p. 93-102, 2011.
\end{abstract}

Resumo: Os conjuntos de artefatos líticos recuperados em distintos tipos de sítios arqueológicos do Chubut mostram uma proeminência de matériasprimas locais, especialmente sílex, xilopalas e vulcanitas, nessa ordem. Esses materiais são acessíveis nas redondezas dos mesmos. As obsidianas, porém, aparecem em reduzida frequência e não se acham nas jazidas locais. $O$ fato de haver fontes de obsidiana caracterizadas geo-quimicamente e localizadas pontualmente no espaço patagônico, possibilitou a identificação da origem dos achados nos resultados das análises geo-químicas de obsidianas de vários sítios no interior de Chubut e são discutidos em relação aos circuitos de mobilidade dos grupos humanos no espaço, estruturado pelas estradas. As conclusões apontam não apenas a existência de corredores de comunicação, mas também de encruzilhadas, ao mesmo tempo em que se propõe o uso da obsidiana associado a aspectos sociais ou simbólicos, e não somente tecnológicos.

Palavras-chave: Patagônia - Obsidianas - Caçadores-coletores - Mobilidade.

\section{Introducción}

n los sitios arqueológicos conocidos
hasta el momento en el interior de

(*) Consejo Nacional de Investigaciones Científicas y Técnicas (CONICET). Instituto Nacional de Antropología y Pensamiento Latinoamericano (INAPL).

<analiacastro@gmail.com>

$\left.{ }^{(* *}\right)$ Consejo Nacional de Investigaciones Científicas y Técnicas
Chubut, los artefactos líticos muestran un alto porcentaje de uso de materias primas locales, especialmente sílices y xilópalos, y en menor medida vulcanitas. Esto estaría señalando un

(CONICET). Universidad de Buenos Aires (UBA). INAPL. <c.perezdemicou@yahoo.com.ar>

$\left.{ }^{* * *}\right)$ Departamento de Ciencias Geológicas, Universidad de Colorado.<Charles.Stern@Colorado.EDU> 
énfasis en el aprovisionamiento local de rocas. Estas materias primas locales se presentan en general, aunque con algunas diferencias en cada región, con una alta disponibilidad en el ambiente, ya que frecuentemente se hallan en abundancia y son de excelente calidad para la talla. Sin embargo, llama la atención que en todas las regiones estudiadas se encuentran, aunque en baja frecuencia, artefactos de obsidiana (instrumentos, desechos y núcleos), materia prima cuyas fuentes son extra regionales. La presencia de esta roca no local involucra la aplicación de estrategias distintas para su obtención y que implicarían una mayor complejidad e inversión de trabajo, sea por su transporte, o por las relaciones de intercambio que deben ponerse en juego para su adquisición. La cuestión es entender el porqué de la decisión de realizar esa inversión extra, siendo las materias locales suficientes, económicamente hablando. De esto se desprende que el origen de este comportamiento debería analizarse dentro de una problemática más bien social (y tal vez simbólica) que económica.

La interacción con otros grupos a lo largo de los distintos momentos en la historia y prehistoria de la región es un aspecto fundamental para la comprensión de la dinámica del uso del espacio. Esta interacción incluiría posibles relaciones de alianza, de hostilidad, de comercio y/o rituales que incidirían en los movimientos anuales de los distintos grupos sociales. El análisis de artefactos confeccionados en obsidiana y la localización de sus fuentes de proveniencia ha demostrado ser un eficiente indicador arqueológico para definir posibles esferas de interacción entre grupos (entre otros Yacobaccio et al. 2002 y 2004; Durán et al. 2004; Carballo et al. 2007; Giesso et al. 2008). La ventaja de tener fuentes de obsidiana caracterizadas geoquímicamente y ubicadas puntualmente en el espacio patagónico, posibilitó la adscripción del origen de las halladas en los contextos arqueológicos. En este trabajo se presentan los resultados de los análisis geoquímicos de obsidianas de varios sitios en el interior de Chubut y se discuten en relación a los circuitos de movilidad de los grupos humanos en el espacio.

\section{La distribución de fuentes y artefactos de obsi- diana en la Provincia de Chubut}

Los análisis geoquímicos realizados en muestras de artefactos arqueológicos de sitios de la región y de nódulos provenientes de dos fuentes de obsidiana localizadas en la zona, muestran un panorama preliminar de la manera en que habría circulado esta materia prima en la provincia de Chubut a lo largo de los últimos 3200 años de ocupación de la región, época en la que se han fechado los contextos con presencia de obsidiana más tempranos para la zona de Piedra Parada (Bellelli 1988; Perez de Micou et al. 1992)

Los artefactos de obsidiana que han sido analizados hasta el momento provienen de sitios emplazados en distintas regiones de la provincia: zona oeste y central (Fig. 1; Stern et al. 2000 y 2007; Bellelli y Pereyra 2002; Stern 2004; Bellelli et al. 2006; Pérez de Micou y Castro 2007) y sitios en la costa (Gómez Otero y Stern 2005). Estos trabajos hablan, en general, de una escasa presencia de materiales de obsidiana en relación con los artefactos confeccionados en otras materias primas líticas locales que presentan una alta disponibilidad en cuanto a su distribución, abundancia y calidad (Bellelli 2005, Gómez Otero y Stern 2005).

Hasta 2005 (Stern et al. 2000 y 2007 (2005); Bellelli y Pereyra 2002; Stern 2004; Gómez Otero y Stern 2005) se había determinado que la totalidad de las muestras analizadas estaban confeccionadas en ocho tipos de obsidiana distintos. De estos ocho tipos sólo se conocía la proveniencia de tres, cuyas fuentes están puntualmente identificadas (Fig. 1). De estas tres fuentes, dos son de la región norte de la provincia de Chubut (SI de Sacanana y T/SCI de Telsen) y una es del noroeste de la provincia de Santa Cruz (PDA de Pampa del Asador) (Gómez Otero y Stern 2005). Los otros cinco tipos de obsidiana de fuente desconocida eran la Desconocida X (hallada en sitios de Piedra Parada); la Desconocida Y (hallada en sitios de Cholila); la Desconocida Z o tipo Cerro Castillo (CC?) (hallada en Cerro Castillo, Piedra Parada y sitios de la Costa); y las T/SCII y MSI (en sitios de la costa). 


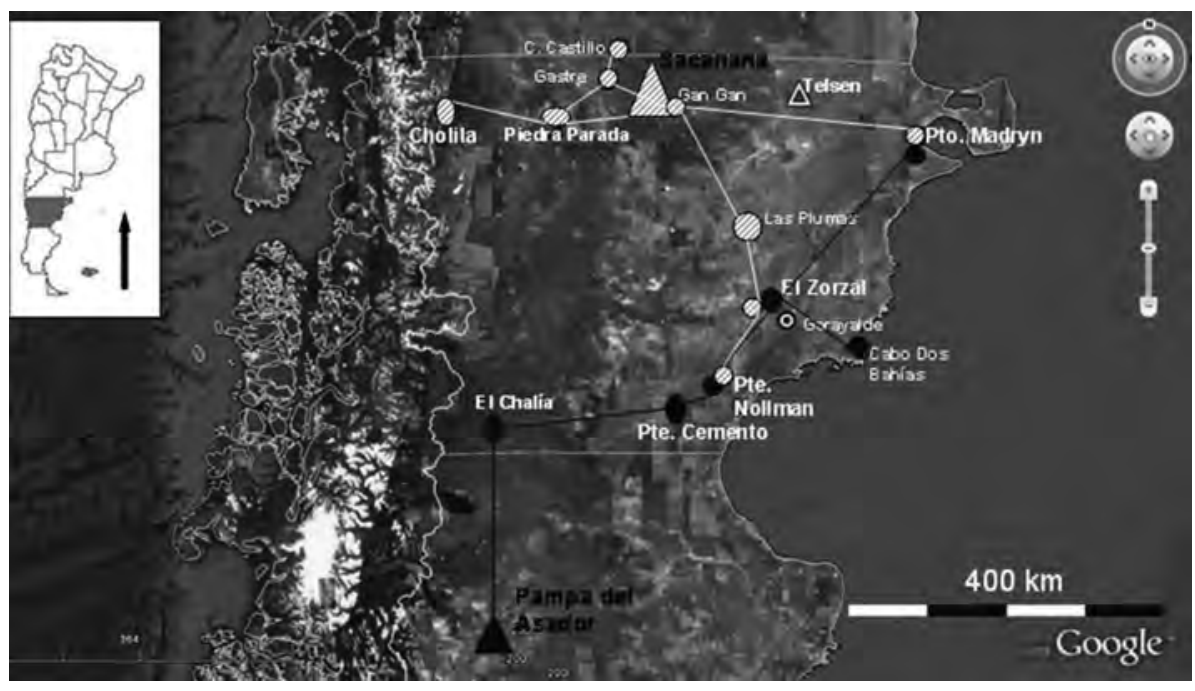

Fig. 1. Ubicación de las principales fuentes de obsidiana: Sacanana y Pampa del Asador; y su distribución en los principales sitios y localidades mencionados.

En el 2006 Bellelli et al. agregan más información a este panorama. Presentan artefactos de obsidiana encontrados en Cholila cuya fuente determinan en Portada Covunco, ubicada en Río Negro. Este tipo de obsidiana era el que habían denominado "Desconocida Y". Por otra parte, hablan de una nueva fuente de obsidiana ubicada en el Parque Nacional los Alerces, en Laguna La Larga, cuya calidad para la talla es mala pero que habría sido utilizada localmente. Estos mismos autores sostienen que la llamada hasta el momento "Desconocida X" proviene de Angostura Blanca, ubicada en Piedra Parada, por lo que pasan a llamar a este tipo de obsidiana como el tipo Angostura Blanca (Bellelli et al. 2006).

Aunque no se conozca aún la proveniencia de las fuentes de algunos de los tipos restantes, su distribución aporta información relevante sobre la manera en que circulaban en el pasado. En líneas generales se observa que el tipo de obsidiana con mayor circulación en la provincia es el tipo SI de la fuente Sacanana, ubicada al sur de la meseta de Somuncura. Hasta el momento, esta obsidiana ha sido hallada en artefactos tanto de la zona de ecotono bosque/estepa (en la localidad de Cholila) (Bellelli y Pereyra 2002; Bellelli et al. 2006), en la estepa en el área de Piedra Parada (Bellelli y Pereyra 2002; Bellelli et al. 2006; Stern et al. 2007), en sitios de Gan Gan y Gastre (Stern et al. 2007; Pérez de Micou y Castro 2007), en la zona central de Chubut en Los Altares y Las Plumas (Stern et al. 2000) y en la costa atlántica (Gómez Otero y Stern 2005; Favier Dubois et al. 2009).

El otro tipo que habría tenido amplia circulación en la región, pero en menor medida que la SI, es la del tipo CC? o Desconocida Z, hallada en artefactos de Cerro Castillo ubicado en el límite oeste de la meseta de Somuncura (Stern et al. 2000), en sitios de Gan Gan (Pérez de Micou y Castro 2007), en Piedra Parada (Stern et al. 2007), y en sitios de la costa (Gómez Otero y Stern 2005).

Por otra parte, algunos tipos de obsidiana se encuentran más circunscriptos, como por ejemplo, las de Laguna La Larga y Portada Covunco que en Chubut sólo aparecen en sitios de Cholila; la obsidiana Desconocida X o Angostura Blanca que sólo aparece en sitios de Piedra Parada; y las obsidianas de Telsen (T/SCI y T/SCII) y MSI que sólo aparecen en sitios de la Costa.

Finalmente, en la costa se destaca la presencia de obsidiana de la fuente de Pampa del Asador en sitios de Puerto Madryn y Cabo Dos Bahías (Gómez Otero y Stern 2005). Estos datos muestran que este tipo de obsidiana habría 
recorrido una distancia aproximada de $800 \mathrm{~km}$ desde su origen.

Con respecto a la cronología, ya se ha mencionado que las obsidianas de Piedra Parada se presentan desde contextos fechados a partir del 3200 AP (Bellelli 1988; Pérez de Micou et al. 1992). En cambio, comenzaría a utilizarse más tarde en los sitios de la costa (2600 AP) (Gómez Otero y Stern 2005) y en Cholila (1900 AP) (Bellelli et al. 2003).

A partir de estos datos se observa un absoluto vacío de información en lo que respecta a las obsidianas de sitios del interior en la zona sur de la provincia. Se presentan aquí datos que vienen a llenar este vacío con información de sitios de la Colonia El Chalía y del Río Chico, ubicados en ambientes de estepa al suroeste y sureste de la provincia. Por otra parte, se suma información para el área de Piedra Parada, especialmente en lo que respecta a los sitios de Oses y Paso del Burro.

Las muestras de obsidiana de estas áreas fueron molidas hasta lograr un polvo fino que fue disuelto en una solución diluida de HF. Con el objetivo de determinar la concentración, en partes por millón (ppm), de los elementos presentes a nivel de trazas en la obsidiana, se analizó la mencionada solución en el Laboratorio de Geología Ambiental de la Universidad de Colorado. Esta fue analizada utilizando técnicas estándar de ICP-MS. Se controló la exactitud y la precisión del ensayo utilizando materiales de referencia internacionales. Para estimar la exactitud se analizaron patrones del laboratorio contrastándolos con estos materiales de referencia internacionales. La precisión se estimó midiendo repetidamente estos patrones y su valor es del $10 \%$. Los elementos traza presentes en las obsidianas analizadas fueron comparados con las composiciones conocidas de los distintos tipos de obsidiana ya descriptas para la región (Fig. 2) para determinar su fuente de proveniencia. Dado que los distintos tipos relevantes de Chubut presentan composiciones muy diferentes, la asignación de las muestras a alguno de los tipos conocidos no dio lugar a ambigüedades.

Se comenzará por hacer mención a las obsidianas de los sitios del norte y luego se presentarán los datos del sur.

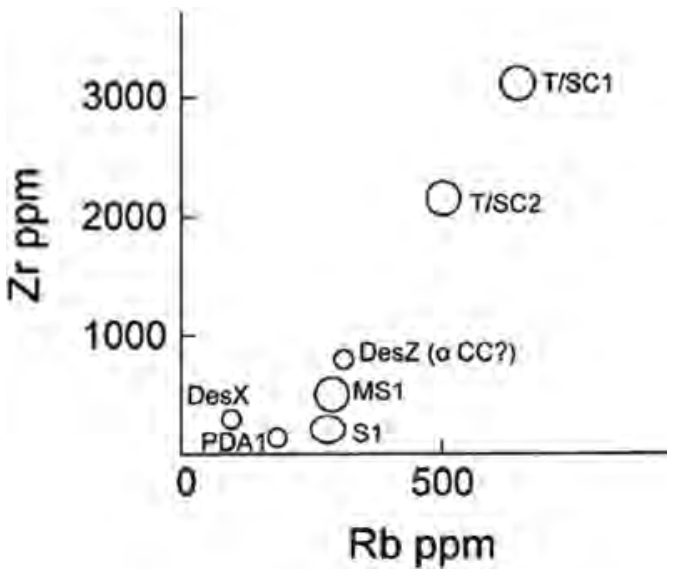

Fig. 2. Concentraciones de $\mathrm{Zr}$ vs. $\mathrm{Rb}$ (en partes de millón) presente en los tipos más importantes de obsidiana de Chubut, basado en los los datos presentados en Stern et al. (2000 y 2005), Bellelli y Pereyra (2002), Stern (2004), Gómez Otero y Stern (2005) y Bellelli et al. (2006). Cada uno de estos distintos tipos son fácilmente distinguibles químicamente únicamente sobre la base de su contenido de $\mathrm{Zr}$ y $\mathrm{Rb}$ y es en base a este tipo de datos sobre las que se han establecido los distintos tipos de obsidiana presentados en las tablas anteriores.

Las Obsidianas del río Chubut medio y de la ruta Gan Gan-Gastre, norte de la Provincia de Chubut

Los trabajos realizados en el extremo este del área de Piedra Parada, curso medio del río Chubut, en los sitios de la Estancia San Ramón (costa sur del Río Chubut), Puesto San Ramón (en las pampas altas) (Castro 2005; Pérez de Micou y Castro 2005; Castro 2007) y los realizados en la zona de Gastre y Gan Gan (Pérez de Micou y Castro 2007), han aportado nuevas evidencias al estado de conocimiento sobre la distribución de obsidianas en la región. Se presentan aquí (Tabla 1) los tipos de obsidiana hallados en estos sitios, y luego se agregan los resultados de nuevas muestras analizadas para los sitios de Oses y Paso del Burro.

El tipo predominante en todas las muestras es el SI de la fuente Sacanana (Fig. 1). La única pieza analizada proveniente de los campos altos de la estancia San Ramón (el Puesto San Ramón) presenta el tipo de obsidiana cuya fuente es la denominada "Desconocida X" o Angostura Blanca (Bellelli et al. 2006) que sería local. Un ejemplar de este tipo de obsidiana también está 
Tabla 1

\begin{tabular}{|c|c|c|}
\hline $\begin{array}{r}\text { Muestras de obsidi } \\
\text { este del área de } \mathrm{P} \\
\text { del río Chub } \\
\text { (Stern et al. } 200\end{array}$ & $\begin{array}{l}\text { nas analiza } \\
\text { dra Parad } \\
\text { t, Gastre y } \\
\text { y Pérez y }\end{array}$ & $\begin{array}{l}\text { as del extremo } \\
\text { curso medio } \\
\text { San Gan } \\
\text { astro 2007) }\end{array}$ \\
\hline Sitio & $\begin{array}{c}\text { Muestra } \\
\mathbf{n}^{\circ}\end{array}$ & $\begin{array}{c}\text { Tipo de } \\
\text { obsidiana }\end{array}$ \\
\hline & CM8 & SI (sacanana) \\
\hline & CM9 & SI (sacanana) \\
\hline & CM10 & SI (sacanana) \\
\hline San Ramón Costa & CM11 & SI (sacanana) \\
\hline & CM12 & SI (sacanana) \\
\hline & CM13 & $\begin{array}{c}\text { Desc X } \\
\text { Angost.Blanca }\end{array}$ \\
\hline Puesto S. Ramón & CM1 & $\begin{array}{c}\text { Desc X } \\
\text { Angost.Blanca }\end{array}$ \\
\hline & $\mathrm{CM} 2$ & SI (sacanana) \\
\hline & $\mathrm{CM} 3$ & SI (sacanana) \\
\hline & CM5 & SI (sacanana) \\
\hline 18 & CM6 & SI (sacanana) \\
\hline & CM7 & SI (sacanana) \\
\hline & $\mathrm{CM} 4$ & Desc $Z_{\text {o }}$ CC? \\
\hline
\end{tabular}

presente en la Estancia San Ramón ubicada en el valle del Río Chubut. Todo el material proveniente de Gastre es del tipo SI de Sacanana. La muestra de Gan Gan presenta cinco artefactos del tipo SI de Sacanana y un artefacto del tipo Desconocida Z o CC?

En la Tabla 2 se presentan los nuevos análisis realizados en las obsidianas de campo Oses (Tapera y Médanos) y del sitio Paso del Burro de la costa de San Ramón. Se observa que la totalidad de las muestras de Oses (nueve artefactos) provienen de la fuente Sacanana. En cambio, las tres muestras analizadas de Paso del Burro, provienen de tres fuentes distintas: una de Sacanana, otra del tipo CC? o Desconocida Z, y la última del tipo Desconocida X (la posible fuente local de Angostura Blanca).

En síntesis, los nuevos datos aportados presentan también a la fuente de Sacanana, ubicada a una distancia de aproximadamente 120 $\mathrm{km}$ de estos sitios, en línea recta, como la más utilizada para los sitios de la orilla norte del río Chubut. Con respecto a los datos de las costa
Tabla 2

Nuevos Análisis realizados para obsidianas de Oses y Paso del Burro- Área Piedra Parada, valle medio del Río Chubut

\begin{tabular}{ccc}
\hline Sitio & $\begin{array}{c}\text { Muestra } \\
\mathbf{n}^{\mathbf{0}}\end{array}$ & $\begin{array}{c}\text { Tipo de } \\
\text { obsidiana }\end{array}$ \\
\hline \multirow{2}{*}{ Tapera Oses } & CS526 & S1-Sacanana \\
& CS528 & S1-Sacanana \\
\hline & CS527 & S1-Sacanana \\
& CS529 & S1-Sacanana \\
Médanos Oses & CS531 & S1-Sacanana \\
& CS532 & S1-Sacanana \\
& CS533 & S1-Sacanana \\
& CS534 & S1-Sacanana \\
& CS535 & S1-Sacanana \\
\hline Costa SR- & CS507 & CC? Desc. Z \\
Paso del Burro & CS709 & SI (sacanana) \\
& CS710 & Desc. X \\
& Ang. B lanca \\
\hline
\end{tabular}

sur, se observa en Paso del Burro una mayor variedad de tipos que incluyen los de Sacanana, Desconocida X, y Desconocida Z o CC?, que ya aparecían en otros sitios de la Estancia San Ramón.

Las obsidianas del sur de la provincia de Chubut:

\section{Colonia El Chalía}

Se analizaron quince muestras provenientes de dos sitios ubicados en la Colonia El Chalía: cinco muestras de Tapera Almeida y diez muestras de Laguna Quilchamal (Tabla 3). En Laguna Quilchamal, se observó que la frecuencia de aparición de artefactos de obsidiana era mayor que en Almeida y también mayor a lo encontrado en los sitios del área del valle medio del Chubut (en estos sitios la frecuencia de obsidianas en artefactos formatizados era del 6,7\%, en los desechos del 5,8\% y en los núcleos $5 \%$, siendo los sitios de Oses los que presentaban los mayores porcentajes). En el Chalía la obsidiana se presenta en un $9,2 \%$ de los instrumentos (en Laguna Quilchamal un 12,2\%), 10,4\% de 
los desechos (19,6\% en Quilchamal) y $8,3 \%$ de los núcleos (13,3\% en Quilchamal). Es decir en la zona del sudoeste del Chubut el uso de la obsidiana habría sido más frecuente que para el noroeste.

\section{Tabla 3}

Resultados de los análisis de obsidianas de Colonia El Chalía, suroeste de la Provincia de Chubut

\begin{tabular}{ccc}
\hline \hline Sitio & $\begin{array}{c}\text { Muestra } \\
\mathbf{n}^{\mathbf{o}}\end{array}$ & $\begin{array}{c}\text { Tipo de } \\
\text { obsidiana }\end{array}$ \\
\hline \multirow{4}{*}{ Tapera Almeida } & CS515 & Desconocido \\
& CS523 & PDA1 - Pampa Asador \\
& CS520 & PDA1 - Pampa Asador \\
& CS525 & PDAIII?- Pampa Asador \\
CS701 & PDA1 - Pampa Asador \\
\hline \multirow{4}{*}{ Lag } & CS 516 & PDA1 - Pampa Asador \\
& CS 517 & PDA1 - Pampa Asador \\
& CS 518 & PDA1 - Pampa Asador \\
CS 521 & PDA1 - Pampa Asador \\
CS 522 & PDA1 - Pampa Asador \\
CS 524 & Desconocido \\
CS702 & PDA1 - Pampa Asador \\
CS705 & PDA1 - Pampa Asador \\
CS706 & PDA1 - Pampa Asador \\
CS707 & PDA1 - Pampa Asador \\
\hline
\end{tabular}

Se observa que la totalidad de las muestras analizadas, salvo dos no identificadas, provienen de la fuente de Pampa del Asador ubicada en la Provincia de Santa Cruz, aproximadamente a $250 \mathrm{~km}$ en línea recta hacia el sur de esta región. Además, en estos sitios está completamente ausente tanto el tipo de Sacanana como los otros tipos hallados en noroeste de la provincia.

\section{El Río Chico}

En los diversos sitios del Río Chico se destacó la bajísima frecuencia de ocurrencia de obsidianas. En la amplia muestra de material lítico obtenida en estos sitios, se hallaron tan sólo seis artefactos de esta materia prima. Cinco de estos seis artefactos pudieron ser analizados geoquímicamente (Tabla 4). A pesar de ser una muestra tan pequeña, aporta resultados muy interesantes para el tema de los circuitos de movilidad, ya que en estos sitios se observa la presencia tanto de obsidiana del tipo Sacanana - muy frecuente en el norte de Chubut pero ausente en el sudoeste - como el de Pampa del Asador - frecuente en el sudoeste pero ausente en el noroeste -.

Tabla 4

Resultados de los análisis de obsidianas del Río Chico

\begin{tabular}{|c|c|c|}
\hline Sitio & $\begin{array}{c}\text { Muestra } \\
n^{\circ}\end{array}$ & $\begin{array}{c}\text { Tipo de } \\
\text { obsidiana }\end{array}$ \\
\hline $\begin{array}{l}\text { La Bajadita- } \\
\text { Pte. Cemento }\end{array}$ & CS511 & $\begin{array}{c}\text { PDA1- } \\
\text { Pampa Asador }\end{array}$ \\
\hline $\begin{array}{l}\text { Puente Nollman- } \\
\text { Transecta }\end{array}$ & CS510 & $\begin{array}{c}\text { PDA1- } \\
\text { Pampa Asador }\end{array}$ \\
\hline $\begin{array}{l}\text { Puente Nollman- } \\
\text { El Tragüil }\end{array}$ & CS514 & S1-Sacanana \\
\hline $\begin{array}{c}\text { Puesto El } \\
\text { ZorzalConc. } 2\end{array}$ & CS512 & S1-Sacanana \\
\hline $\begin{array}{c}\text { Puesto El } \\
\text { Zorzal-Conc. } 2\end{array}$ & CS513 & $\begin{array}{c}\text { PDA1- } \\
\text { Pampa Asador }\end{array}$ \\
\hline
\end{tabular}

Las obsidianas de Pampa del Asador se encuentran en artefactos de La Bajadita (a 330km aproximados en línea recta de esa fuente); en Puente Nollman (a $390 \mathrm{~km}$ aprox. en línea recta de la fuente); y en Puesto El Zorzal (el más alejado de la fuente, a $500 \mathrm{~km}$ aprox. en línea recta). Las de Sacanana se hallan en El Tragüil en Puente Nollman (a $300 \mathrm{~km}$ aprox. en línea recta del Cerro Guacho), y en El Zorzal (a $250 \mathrm{~km}$ aprox. de la fuente en una línea recta por la ruta hacia las Plumas).

\section{Síntesis y discusión}

Hasta ahora los resultados de los estudios realizados en el interior de Chubut muestran una alta disponibilidad local de recursos líticos en cuanto a calidad, distribución y abundancia, y plantean la falta de necesidad de aplicar estrategias logísticas para la búsqueda de materias primas alóctonas, ya que las disponibles son apropiadas para la confección de todos los grupos tipológicos. Esto podría explicar la escasez generalizada de 
obsidiana, a pesar de que, en general, hablamos de distancias moderadas desde las fuentes conocidas en Chubut hasta los sitios arqueológicos en donde se hallan los artefactos (salvo para el caso del Río Chico y Puerto Madryn que presentan distancias mayores con respecto a Pampa del Asador).

Pero más allá de su escasez, la presencia diferencial de tipos de obsidiana en los distintos sitios arqueológicos podría indicar una circulación de bienes direccional y restringida. El tipo SI de la fuente Sacanana está presente en todos los sitios arqueológicos del norte y centro de la región, desde la zona de ecotono bosque/estepa hasta los sitios ubicados en la costa atlántica, y ausente en los sitios del sudoeste de Chubut. Por otra parte, hay otros tipos de obsidiana (T/SI, $\mathrm{T} / \mathrm{SII}$, y MSI) halladas en artefactos provenientes de sitios ubicados en la costa atlántica que no aparecen en sitios hacia el oeste de la provincia.

En cuanto a las evidencias de Gastre y Gan Gan es esperable que, por su cercanía a la fuente Sacanana, se presenten muestras con mayores porcentajes de obsidianas (el 16,7\% de los desechos de talla de la muestra presentada son de obsidiana) y mayoritariamente la del tipo SI. También es importante ver aquí que la amplia distribución de esta fuente puede estar relacionada con su ubicación en esta zona central atravesada por varias rutas indígenas según la información etnohistórica y etnográfica disponible (Pérez de Micou y Castro 2007).

Con respecto al tipo Desconocida X (Angostura Blanca), que hasta el momento sólo fue hallada en la zona de Piedra Parada, es interesante mencionar que sólo se encuentra en los sitios al sur del Río Chubut. Aparece en aquellos ubicados sobre la margen sur del río y hay mayor cantidad de hallazgos de este tipo de obsidiana en los sitios de las pampas de altura del sur (Stern et al. 2007). Estos sitios aparentemente estarían en relación dinámica con los sitios del valle (ver Castro 2005): Campo Nassif 1 en la costa del río Chubut y Bajada del Tigre en los campos altos (Bellelli y Pereyra 2002); y el sitio Estancia San Ramón en el valle y Puesto San Ramón en los campos altos. Por otro lado, es justamente en la costa sur del río en donde se ubica el sitio Angostura Blanca del cual provendría esta obsidiana.
En los sitios del Río Chico se han encontrado muy pocos artefactos de obsidiana pero es destacable la presencia de obsidianas de Sacanana, ya que esta evidencia amplía hacia el sur el rango de dispersión del tipo SI. Por otro lado, es notable que esta obsidiana esté completamente ausente en El Chalía, sudoeste del Chubut, en donde predominan las obsidianas de Pampa del Asador.

A partir de estas evidencias surge una serie de inquietudes: ¿cómo se explica la aparente unidireccionalidad centro-costa de la circulación de las obsidianas en el norte de Chubut, ya que en la costa hay tipos de obsidiana que no circularon hacia el oeste? Del mismo modo, cuál sería la posible explicación de que la obsidiana de Sacanana no haya circulado hacia el sudoeste de la región? ¿Es posible que esta información esté indicando algún tipo de restricción en la circulación de bienes y/o grupos en el interior de la Patagonia? ¿Cuál sería la posible explicación del hecho de que la obsidiana de Pampa del Asador haya llegado hasta sitios tan alejados como la costa norte chubutense y que también se encuentre en el sudoeste de Chubut, en la colonia El Chalía, pero que no se encuentre en el área de Piedra Parada cuya conexión por el interior sería más directa?

Las fuentes etnohistóricas dan cuenta de grupos indígenas circulando por rutas que conectarían perfectamente al noroeste de la Provincia de Santa Cruz con el noroeste de Chubut, por el interior, transitables tanto a caballo como a pie (ver por ejemplo Musters 1964). Por otra parte, no hay datos paleoambientales que señalen fluctuaciones importantes en el ambiente entre los 3200 AP y los momentos históricos (D'Antoni 1978; Burry y D’Antoni 2001; Páez 1990, Garleff et al. 1994, Prieto y Stutz 1996, Whatley y Cusminsky 1999, en Fernández 2006; Trivi y Burry 2007; Burry y Trivi 2009). Además, aparentemente, no habría barreras biogeográficas que impidan esta comunicación, por lo que habría que pensar en posibles "barreras culturales".

$\mathrm{Al}$ ser la obsidiana un recurso que no es imprescindible para la tecnología, ya que hay otras materias primas locales disponibles para usos semejantes, se puede sostener que en la circulación de este tipo de artefactos estarían entrando en juego variables que podrían estar relacionadas con cuestiones sociales vinculadas con intercambios, 
alianzas, o razones de prestigio. En este sentido, la zona del Río Chico -que presenta obsidianas tanto de la fuente de Sacanana como la de Pampa del Asador, y está a mitad de camino entre esta fuente y Puerto Madryn- podría haber funcionado como un corredor que conectaría a las regiones del norte de la provincia con las del sur (incluyendo las áreas costeras; Fig. 1). Las fuentes históricas sustentan esta idea, al menos para el siglo XIX. Es de destacar que el Puesto El Zorzal presenta ambos tipos de obsidiana y que es justamente por allí por donde cruza la ruta Garayalde- Las Plumas. Sobre este camino se ha registrado evidencia aislada de materiales arqueológicos (Castro y Pérez de Micou 2010). Entre ellos no se ha hallado obsidiana, no obstante es importante mencionar el trayecto completo de esta ruta que comenzaría en la costa atlántica, justamente cerca de la zona de Cabo Dos Bahías en donde se ha informado la presencia de obsidiana de Pampa del Asador (Gómez Otero y Stern 2005); luego cruza el río Chico, a la altura del Puesto El Zorzal; de allí continúa hasta las Plumas sobre el río Chubut; y desde las Plumas varios caminos vecinales se dirigen hacia la ruta 11 que conduce hasta Gan Gan (Fig.1). Esto lleva a pensar en una posible conexión entre estas áreas facilitada por este camino. Por otro lado, en el área de Puente Nollman, que es el otro sitio en donde se presentan obsidianas de ambos tipos, también cruza una ruta actual que, atravesando la Pampa Pelada y bordeando la Sierra Cuadrada, llega también hasta el valle del río Chubut en un punto ubicado a $70 \mathrm{~km}$ al oeste de las Plumas. Estos datos llevan a pensar en la posibilidad de que Nollman y El Zorzal hubieran funcionado como encrucijadas (Pérez de Micou y Castro 2007) en donde, al igual que en Gan Gan, confluirían diversos caminos de importancia.

\section{Agradecimientos}

Participaron en los trabajos de campo María Laura Casanueva, María Luz Funes y Mariana Sacchi. Agradecemos especialmente a la familia Oses, a Bruno Nicoletti, a Coca San Martín y a toda la comunidad de El Chalía por su hospitalidad y permanente colaboración.

CASTRO ESNAL, A.; PÉREZ DE MICOU, C.; CHARLES R. STERN, Ch.R.

Obsidian circulation in Chubut, central Patagonia, Argentina: use of extra regional raw materials as a sign of mobility and interaction between hunter gatherers groups.

R. Museu Arq. Etn., São Paulo, n. 21, p. 93-102, 2011.

Abstract: The lithic assemblages recovered from various types of archaeological sites in Chubut, central Patagonia, show a predominance of local raw materials such as silica, xilopals and vulcanite. These materials are available in the vicinities of these sites. Obsidians, however, scarcely appear in archaeological sets and are not found in local quarries. The availability of geochemically characterized obsidian sources, precisely located in the Patagonian area, allowed the identification of the source location of those found in archaeological contexts. This paper presents the results of the geochemical analysis of obsidian artifacts from several sites inside Chubut and they are discussed in relation to the mobility circuits of human groups along structured routes. The conclusions suggest not only the existence of communication corridors but also of crossroads. Finally, the use of obsidians associated with social or symbolic aspects, opposed to a merely technological application, is argued.

Keywords: Central Patagonia - Obsidians - Hunters gatherers - Mobility. 


\section{Referencias bibliográficas}

\section{BELLELLI, C.}

1988 Recursos Minerales: su estrategia de aprovisionamiento en los niveles tempranos de Campo Moncada 2 (Valle de Piedra Parada, Río Chubut). Arqueología Contemporánea Argentina. Búsqueda, Yacobaccio ed: $147-176$

BELLELLI, C.; PEREYRA, F.

2002 Análisis geoquímicos de obsidiana. Distribuciones de fuentes y artefactos arqueológicos en el Noroeste del Chubut (Patagonia Argentina). Revista Werken, 3: 99-118.

BELLELLI, C.; CARBALLIDO, M.; FERNÁNDEZ P.; SHEINSOHN, $\mathrm{V}$.

2003 El pasado entre las hojas. Nueva información arqueológica del noroeste de la Provincia del Chubut, Argentina. Revista Werken, 4: 25-42.

BELLELLI, C.; PEREYRA, F.; CARBALLIDO, M.

2006 Obsidian localization and circulation in northwestern Patagonia (Argentina): sources and archaeological record. In: Maggetti, M.; Messiga, B. (Eds.) Geomaterials in Cultural Heritage. Geological Society of London, Special Publications 257: 421255.

BURRY, L.S.; D'ANTONI, H.

2001 Análisis de polen del sur de Chubut (Argentina): reinterpretación del perfil Alero del Cañadón de Las Manos Pintadas en base a análogos modernos. Asociación Paleontológica Argentina. Publicación Especial 8. XI Simposio Argentino de Paleobotánica y Palinología: 65-72.

BURRY, L.S.; TRIVI de MANDRI, M.

2009 ¿Cómo conocer el ambiente del pasado? El polen como representante de la vegetación. In: Pérez de Micou, C.; Trivi de Mandri, M.; Burry, L.S. (Eds.) Imágenes desde un alero. Investigaciones multidisciplinarias en Río Mayo, Chubut. Patagonia Argentina. Fundación de Historia Natural Félix de Azara. Bs. As: 23-30.

CARBALLO, D.; CARBALLO, J.; NEFF, H.

2007 Formative and Classic Period obsidian procurement in Central Mexico: a compositional study using Laser Ablation-Inductively Coupled Plasma-Mass Spectrometry. Latin American Antiquity, 18 (1): 27-43.
CASTRO, A.

2005 Estancia San Ramón. Estudio de la organización tecnológica en una microregión del área de Piedra Parada, Noroeste de la Provincia de Chubut. Tesis de Licenciatura en Ciencias Antropológicas, orientación Arqueología, Facultad de Filosofía y Letras, Universidad de Buenos Aires.

2007 Estancia San Ramón. Una micro-región del Área de Piedra Parada Chubut. In: Aqui Vivieron. AINA: 43-66.

CASTRO, A.; PÉREZ de MICOU, C.

2010 De Garayalde a Las Plumas. Exploración de un espacio entre dos ríos. In: Bárcena, J.R. ; Chivazza, H. (Eds.) Arqueología argentina en el bicentenario de la revolución de mayo. UNCuyo-CONICET. Mendoza. Tomo V, Cap. 35: 1877-1882.

D'ANTONI, $\mathrm{H}$.

1978 Palinología del Perfil del Alero del Cañadón de las Manos Pintadas (Las Pulgas, Provincia del Chubut). In: Relaciones de la Sociedad Argentina de Antropología Vol. XII; N. S. Bs. As.: 249-263.

DURÁN, V.; GIESSO, M.; GLASCOCK, M.; NEME, G.; GIL, A.; SANHUEZA, L.

2004 Estudio de fuentes de aprovisionamiento y redes de distribución de obsidiana durante el Holoceno tardío en el sur de Mendoza (Argentina). Estudios Atacameños, 28:25-44.

FAVIER DUBOIS, C.; STERN, C.; CARDILLO, M.

2009 Caracterización de la distribución y tipos de obsidiana presentes en la costa rionegrina, Argentina. In: Salemme, M.; Santiago, F.; Álvarez, M.; Piana, E.; Vásquez, M.; Mansur, E. (Eds.) Arqueología de la Patagonia. Una mirada desde el último confín. Ushuaia, Editorial Utopías: 349-359.

FERNÁNDEZ, P.M.

2006 Aprovechamiento de recursos faunísticos en los ambientes de estepa y ecotono bosque-estepa del norte de la provincia del Chubut. Tesis de Doctorado, Facultad de Filosofía y Letras, Universidad de Buenos Aires. MS.

GIESSO, M.; BERÓN, M.; GLASCOCK, M.

2008 Obsidian in Western Pampas: source characterization and provisioning strategies. IAOS Bolletin, 38: 15-18. 
GÓMEZ OTERO, J.; STERN, C.

2005 Circulación, intercambio y uso de obsidianas en la costa de la provincia del Chubut (Patagonia Argentina) durante el Holoceno Tardío. Intersecciones en Antropología, 6. FACSO, UNCPBA: 93-108.

MUSTERS, G.

1964 (1871) Vida entre los patagones. Solar-Hachette. Bs. As.

PEREZ de MICOU, C.B.; BELLELLI, C.; ASCHERO, C.

1992 Vestigios minerales y vegetales en la determinación del territorio de explotación de un sitio. Análisis espacial en la Arqueología Patagónica. Búsqueda de Ayllu S.R.L. Bs. As.: 53-81.

PÉREZ de MICOU, C.; CASTRO, A.

2005 Recuperar un paisaje. Prospecciones en la Estancia San Ramón, Provincia de Chubut. Revista Relaciones, XXX: 263-275

2007 Los caminos indigenas. La ruta Gan-Gan/Gastre (Chubut). In: Aqui Vivieron. Arqueología y ambiente en Patagonia. AINA. Bs. As.: 9-28.

STERN, Ch.R.

2004 Obsidian in Southern Patagonia: review of the current information. In: Civalero, M.T.; Fernández, P.; Guráieb, A. INAPL (Comp.) Contra Viento y Marea. Arqueología de Patagonia, Sociedad Argentina de Antropología: 167-178.

STERN, Ch.R.; GÓMEZ OTERO, J.; BELARDI, J.B.

2000 Características químicas, fuentes potenciales y distribución de diferentes tipos de obsidianas en el Norte de la Provincia del Chubut, Patagonia Argentina. Anales del Instituto de la Patagonia, 28, Punta Arenas, Chile: 275-290.

STERN, Ch.R.; BELLELLI, C.; PÉREZ DE MICOU, C.

2007 Sources and distribution of geologic and archaeologic samples of obsidian from Piedra Parada, North-Central Chubut, Argentine Patagonia. In: Morello, F.; Martinic, M.; Prieto, A.; Bahamonde, G. (Eds.) Arqueología de Fuego-Patagonia. Levantando piedras, desenterrando huesos...y develando arcanos. CEQUA Ed. Punta Arenas, 2005: 205-208.

TRIVI DE MANDRI, M.; BURRY, L.S.

2007 Paleoambientes del Lago Colhue Huapi (Chubut, Argentina) durante el Holoceno reciente. Estudio palinológico. Revista Española de Micropaleontología, 39 (3): 205 214.

YACOBACCIO, H.; ESCOLA, P.; LAZZARI, M.; PEREYRA, F.

2002 Long-distance obsidian traffic in Northwestern Argentina. In: Glascock, M. (Ed.) Geochemical Evidence for Long-Distance Exchange. Scientific Archaeology for the Third Millennium. Wesport, Connecticut, Bergin y Garvey: 167-204.

YACOBACCIO, H.; ESCOLA, P.; PEREYRA, F.; LAZZARI, M.; GLASCOCK, M.D.

2004 Quest for ancient routes: obsidian sourcing research in Northwestern Argentina. Journal of Archaeological Science, 31: 193-204. 